\title{
Dehazing using Fast Guided Image Filtering
}

\author{
P. Swetha, K. Vidhya
}

\begin{abstract}
Single image edge-saving smoothing methods such as guided picture shifting (GIF) and weighted guided picture shifting (WGIF) couldn't save fine structure. In this paper, another all inclusive guided picture shifting GIF is presented to defeat the issue. The Guided image filtering is made out of a worldwide structure exchange channel and a worldwide edgesaving smoothing channel. The proposed channel is connected to ponder single picture fog evaluation. Trial results demonstrate that fine structure of the dehazed picture is in reality protected better by the proposed Guided image filter what's more, the dehazed pictures by the proposed Guided filter are more than those dehazed pictures by the current Guided image filtering. from a nearby direct model, the guided channel figures the shifting yield by considering substance of direction picture. Guided channel be utilized as an edge-saving smoothing administrator like famous respective channel, however it has better practices close edges. In addition, channel normally quick and non approximate direct calculation, paying little mind to the bit size and the force go. Right now, it is one of the quickest edgesaving channels.
\end{abstract}

\section{INTRODUCTION}

The execution of visual exercises, for example, object location and acknowledgment depends vigorously on the recognition of open air regular scenes. The pictures of outside scenes are frequently corrupted in the awful climate, for example, dimness, haze, smoke, downpour, etc. The light is mixed with surrounding light reflected from different headings into the line of locate by barometrical particles. All things considered, the items caught under the terrible climate conditions experience the ill effects of low complexity, black out shading, and moved luminance. Cloudiness expulsion can essentially build the differentiations articles, and right shading mutilation caused on the air light. Accordingly, cloudiness evacuation is profoundly requested in picture handling and PC vision applications. Many single picture cloudiness expulsion calculations were proposed because of their expansive applications. In light of a perception that a dimness free picture has the higher difference than its cloudiness picture, intriguing picture fog evacuation calculation was proposed in by expanding the nearby complexity of the reestablished picture utilizing irregular field. Despite the fact that the

calculation in is ready to accomplish outwardly convincing outcomes, it will in general produce overimmersed pictures which probably won't be physically substantial. The direct model was at long last received to structure a solitary picture cloudiness evacuation calculation with the assistance of the guided picture shifting (GIF) in. Likewise, the fog is expelled well on the off chance that it is

\footnotetext{
Revised Manuscript Received on September 10, 2019.

P. Swetha, Department of Electronics and Communication Engineering, Saveetha School of Engineering, Saveetha Institute of Medical and Technical Sciences, Chennai, Tamilnadu, India.

Dr. K. Vidhya, Department of Electronics and Communication Engineering, Saveetha School of Engineering, Saveetha Institute of
} Medical and Technical Sciences, Chennai, Tamilnadu, India. light. In any case, the nature of the dehazed pictures should be improved if it is overwhelming. Enlivened by a perception in that solitary picture dimness evacuation can be viewed as a sort of spatially differing detail upgrade, a perfect structure was proposed in by presenting a neighborhood edge-protecting smoothing based strategy to appraise the transmission guide of a fog picture. Be that as it may, neighborhood edge-protecting smoothing procedures, for example, the GIF in and the weighted GIF (WGIF) in could over smooth pictures, particularly in territories of fine structure. The GIF in and the WGIF in are received to ponder single picture cloudiness expulsion. In this way, both the Guided image filtering and the WGIF couldn't safeguard the fine structure even in spite of the fact that they are basic. The proposed Guided image filter is motivated by GIF in, the WGIF, and the angle space picture handling calculations in, Two noteworthy goals of the GIF and WGIF: 1) are to exchange the structure of the direction picture to the information picture; and 2) to smooth the exchanged picture to deliver the yield picture. Both the goals are accomplished all the while in the GIF and WGIF. They are accomplished independently in the proposed G-GIF. The proposed channel is made out of a worldwide structure exchange channel and a worldwide edge-saving smoothing channel. Contributions of the structure exchange channel are a picture to be sifted what's more, a direction of vector field. Structure is characterized by direction of vector field and it is exchanged to the picture to be sifted by the structure exchange channel. Not at all like the GIF and WGIF in, the structure exchange channel figured as quadratic advancement issue. The proposed edge-safeguarding smoothing channel is propelled by the weighted least square (WLS) channel in and the detail extraction issue in As Outlined in proposed Guided image filtering the fine structure superior to the GIF and Weighted Guided Image Fitering.

\section{GLOBALLY GUIDED IMAGE FILTERING:}

Guided image filtering in, the WGIF in, the angle area picture handling calculations in, and, the WLS channel in and the quadratic enhancement issue in, another sort of GIFs is proposed in this segment. Not at all like the GIF in and WGIF in, the proposed channel is worldwide channel and it is along these lines called G-GIF. The structure exchange channel is roused by the Guided image filtering in, the WGIF in, and the inclination space picture preparing calculations in, and. The contributions of the structure exchange channel are a picture to be sifted and direction of vector field. The target of the structure exchange channel to exchange structure to picture to be separated. 
From the selecting one image as input image. By using guided image filtering we will take the input image.

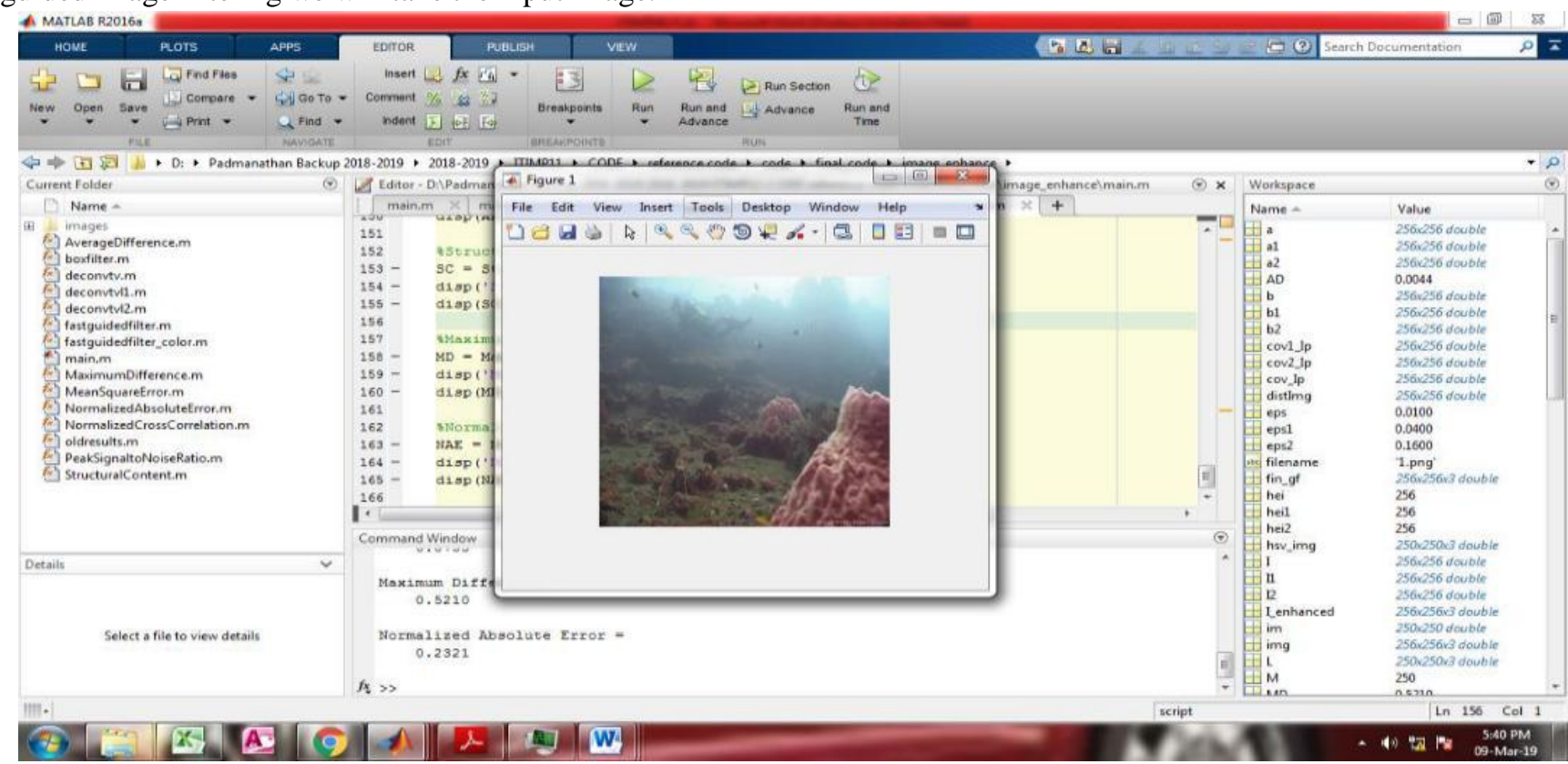

Fig 1: Input image

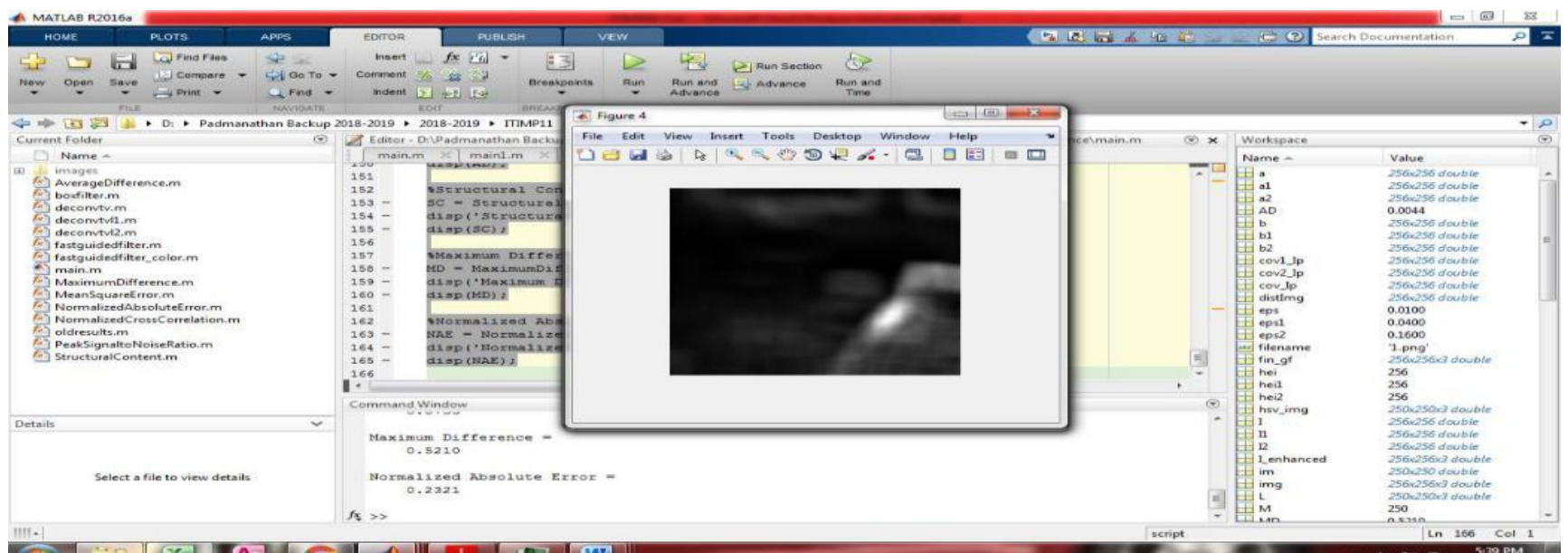

Fig 2:

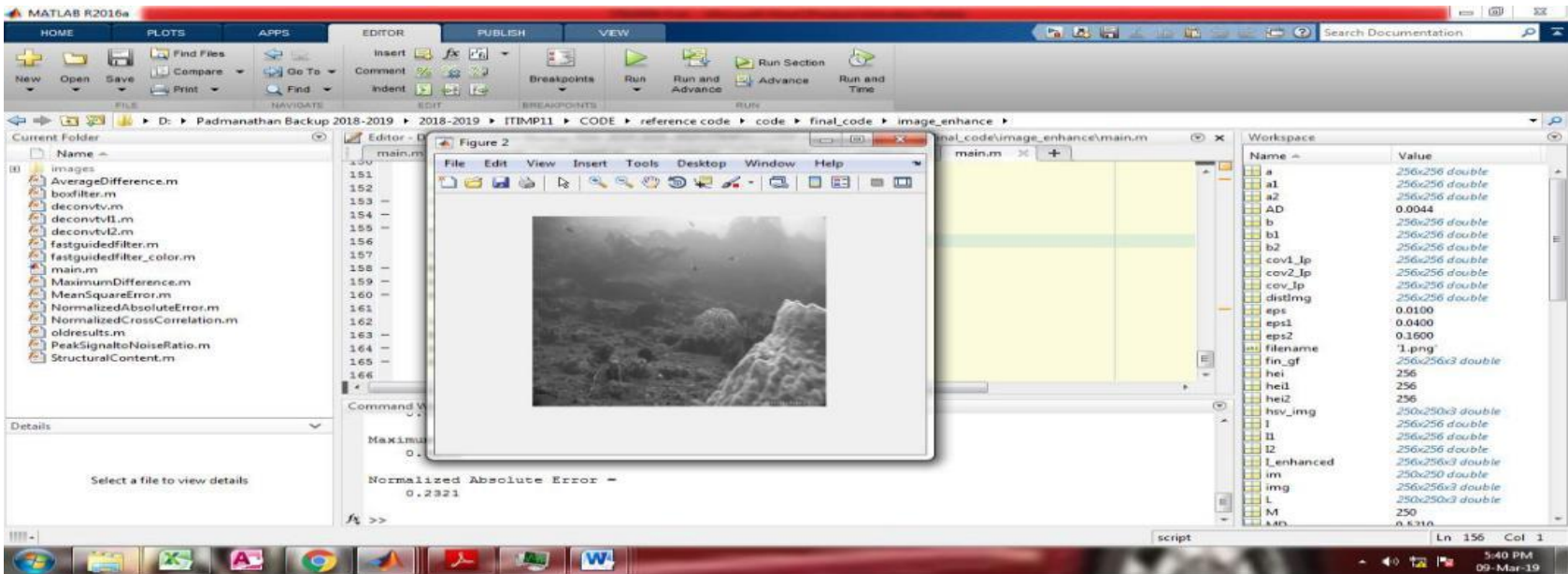

Fig 3: Preprocessed image

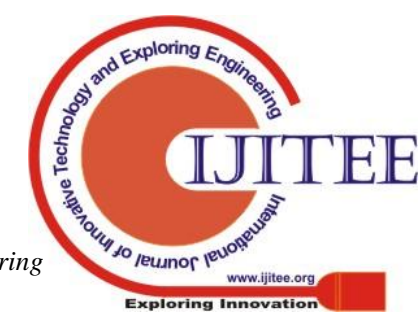




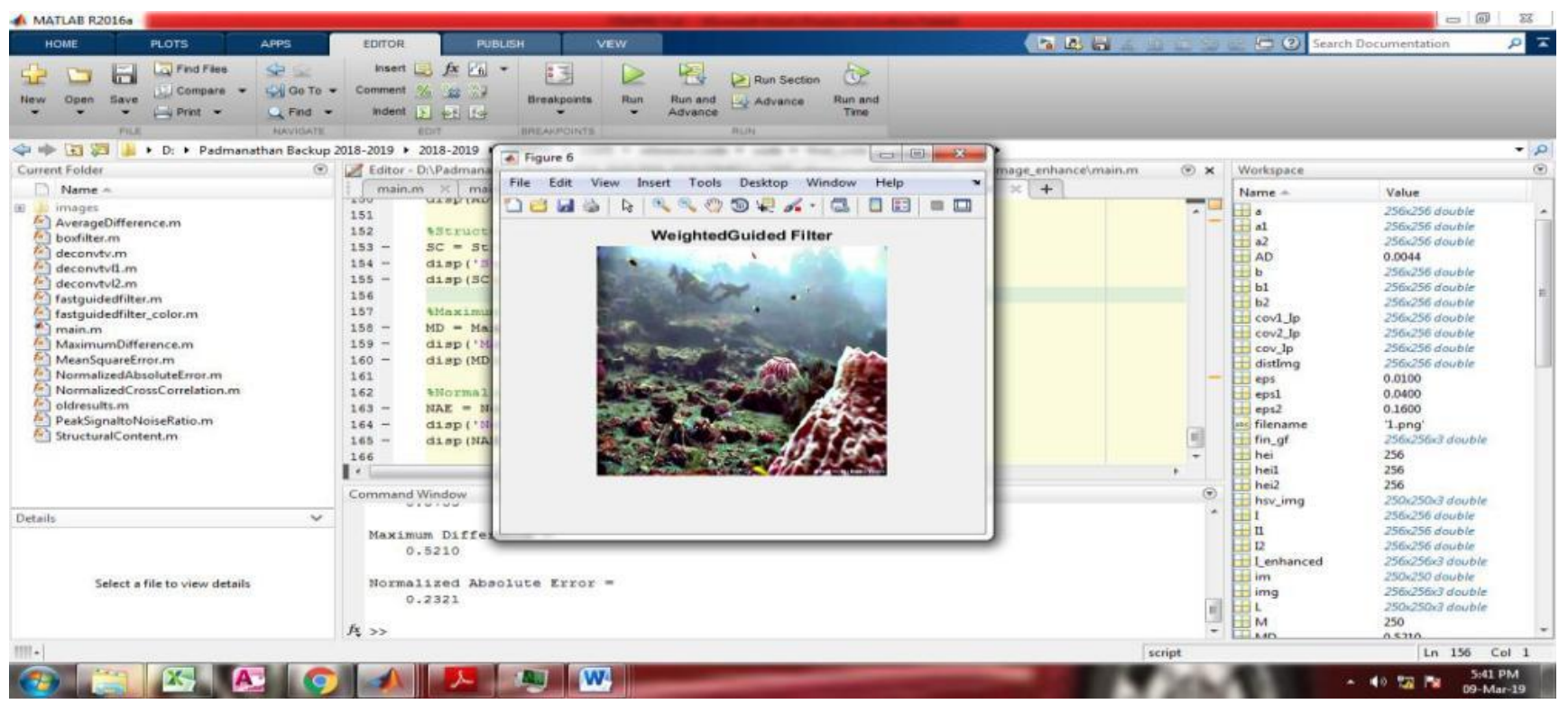

Fig 4:output image

we will remove all fusion and noise by using preprocessor, Boxfilter and globally guided image filtering using all this by one by one step we will get the final output of dehazing image using fast guided image filtering.

\section{EXPERIMENTAL RESULTS:}

In this segment, distinctive decisions of $\lambda$ in the Equation just as $\gamma, \theta$, and in the Equation are first tried. It is appeared in that the proposed G-GIF isn't touchy to the decisions of $\lambda$ in the Equation just as $\gamma, \theta$, and in the Equation. The proposed G-GIF is additionally contrasted and the GIF in also, the WGIF in by utilizing them to examine single picture fog evacuation. It is appeared in Fig. 10 that the proposed G-GIF jelly the structure of branches and leafs better than the GIF and WGIF. It is additionally shown in Fig. 1 that the proposed G-GIF jam the structure of hair of the human subject superior to the GIF and WGIF. Along these lines, the proposed GGIF protects fine structure superior to the GIF and WGIF. The proposed dimness evacuation calculation is then thought about with three cutting edge fog expulsion calculations in, what's more, by testing three fog pictures. The direction picture is chosen as the insignificant shading channel of the fog picture for every one of the calculations in, and. Every one of the settings of the parameters are chosen by the calculations in, and . It is shown in that the calculation in evacuates dimness superior to other fog expulsion calculations yet it enhances clamor in most brilliant areas.

\section{PROPOSED SYSTEM}

Single picture fog evacuation is the under-compelled, on account of the quantity of opportunities is bigger than the quantity of perceptions contains. In the specific, weighted guided picture channel is received to the break down disentangled dim channel. Transmission guide expected from the base layer, and it connected reestablish cloudiness picture.

\section{FUTURE SCOPE}

Nearby edge-saving smoothing strategies, for example, guided picture sifting (GIF) and weighted guided picture separating (WGIF) couldn't save fine structure. In this paper, another internationally guided picture separating (G-GIF) is acquainted with defeat the issue. The proposed channel is connected to concentrate single picture fog evacuation. Trial results demonstrate that fine structure of the dehazed picture is to be sure safeguarded better by the proposed G-GIF and the dehazed pictures by the proposed G-GIF are more honed than those dehazed pictures by the current GIF.

\section{CONCLUSION AND DISCUSSION:}

Another internationally guided picture separating is presented in this paper. The proposed channel can be connected to create more keen pictures and jelly subtleties in areas of fine structure noticeably superior to anything the current privately guided picture separating. It is connected to contemplate single picture murkiness expulsion. Trial results exhibit that the proposed dimness expulsion calculation in fact improves visual nature of dehazed pictures. Other than single picture dimness evacuation, there are numerous uses of the proposed channels. For instance, the channel can be connected to consider scene imaging, edge-mindful smoothing pyramid for introduction combination, detail improvement, picture tangling, HDR pressure, feathering, high goals up-testing, etc.

\section{REFERENCES}

1) A. C. Bovik, "Referenceless expectation of perceptual haze thickness and perceptual picture defogging,". Picture Process. 
2) R. Tan, "Perceivability in terrible climate from a solitary picture," in Proc. IEEE Conf. Comput. Vis.

3) R. Fattal, "Single picture dehazing," in Proc. SIGGRAPH, New York, NY, USA, Jun. 2008, pp. 1-9.

4) P. S. Chavez, "An improved dull article subtraction procedure for climatic dissipating revision of multispectral information," Remote Sens. Environ., vol. 24, no. 3, pp. 459-479, Apr. 1988.

5) F. Kou, W. Chen, C. Wen, and Z. Li, "Slope area guided picture sifting," IEEE

6) Trans. Picture Process., vol. 24, no. 11, pp. 4528-4539, Nov. 2015.

7) Z. Li and J. Zheng, "Edge-preserving decompositionbased single image haze removal,".

8) J. Park, H. Kim, Y. W. Tai, M. S. Brown, and I. Kweon, "High quality depth map upsampling for 3D-TOF cameras," in Proc. IEEE Int. Conf. Comput. Vis., Colorado Springs, CO, USA, Jun. 2011, pp. 1623-1630.

9) M. Werman, "Gradient domain high dynamic range compression," ACM Trans. Graph.

10) E. S. L. Gastal, "Domain transform for edgeaware image and video processing," ACM Trans. Graph.

11) R. Szeliski, "Edge-preserving decompositions for multiscale tone and detail manipulation,". 